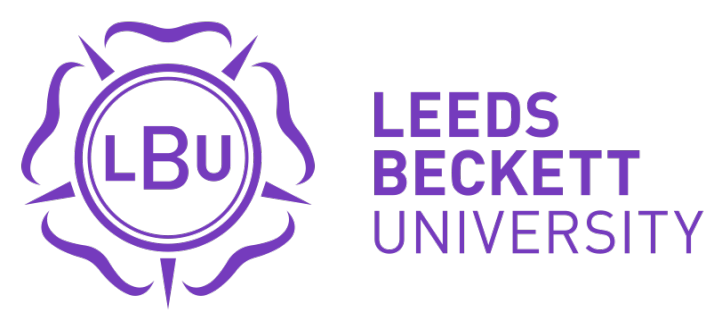

Citation:

Bagheri Zadeh, P and Sheikh Akbari, A (2015) Evaluation of Wavelet Transform Families in Image Resolution Enhancement. In: 2nd IET International Conference on Intelligent Signal Processing 2015 (ISP). IET. ISBN 978-1-78561-136-0

Link to Leeds Beckett Repository record:

https://eprints.leedsbeckett.ac.uk/id/eprint/1817/

Document Version:

Book Section (Accepted Version)

The aim of the Leeds Beckett Repository is to provide open access to our research, as required by funder policies and permitted by publishers and copyright law.

The Leeds Beckett repository holds a wide range of publications, each of which has been checked for copyright and the relevant embargo period has been applied by the Research Services team.

We operate on a standard take-down policy. If you are the author or publisher of an output and you would like it removed from the repository, please contact us and we will investigate on a case-by-case basis.

Each thesis in the repository has been cleared where necessary by the author for third party copyright. If you would like a thesis to be removed from the repository or believe there is an issue with copyright, please contact us on openaccess@leedsbeckett.ac.uk and we will investigate on a case-by-case basis. 


\title{
Evaluation of Wavelet Transform Families in Image Resolution Enhancement
}

\author{
P Bagheri Zadeh*, A Sheikh Akbari ${ }^{\dagger}$ \\ *School of Computer Science and Informatics, De Montfort University, U.K., Pooneh.bagherizadeh@dmu.ac.uk, \\ ${ }^{\dagger}$ School of Computing, Creative Technology and Engineering, Faculty of Arts, Environment and Technology, Leeds Beckett \\ University, U.K., a.sheikh-akbari@leedsbeckett.ac.uk
}

Keywords: Image resolution enhancement, wavelet transform, cycle spinning.

\begin{abstract}
Wavelet transform have been used in many image enlargement techniques and shows superior results compared to conventional image resolution enhancement techniques. However, the choice of wavelets for image enlargement has not been fully investigated. This paper presents an investigation on the performance of a generic wavelet based image enlargement technique, with respect to the choice of wavelets. A Wavelet based image enlargement technique using cycle-spinning is employed for the purpose of this investigation. Different wavelet transforms from wavelet transform families such as Daubechies, symlet, Coiflet, Biorthogonal, Reverse Biorthogonal and Discrete Meyer wavelets are used in this investigation. A summary of the process for this investigation is as follows: A high resolution image is first generated from an input low resolution image, using an inverse wavelet transform, where the coefficients in high frequency subbands are sets to zero. The cycle spinning method is then used to remove the ringing artefacts of the resulting high-resolution image. The generated results indicate that the performance of the image enlargement technique is a function of the chosen wavelet and also the frequency components or texture of the image. Results implies that the enlargement method produces superior results using Daubechi 9/7 and Symlet 2 wavelets, when enlarging images containing significantly higher frequency components. The technique generates superior results when dealing with images containing lower high frequency components using Daubechi 9/7 and LeGall 5/3.
\end{abstract}

\section{Introduction}

Image resolution enhancement is a widespread topic in the field of image processing. Interpolation methods are the widely used techniques for constructing higher resolution image from a lower resolution image while maintain the image quality. The four well-known conventional image interpolation techniques are the nearest-neighbour, bilinear, bicubic and Sinc. The nearest-neighbor interpolation method assigns the intensity of its nearest neighbour in the original image to each new location. In the bilinear interpolation, four nearest neighbours are used to estimate the intensity value of a given location. The bicubic interpolation uses sixteen nearest neighbours to estimate the intensity value of a given location [1]. The Sinc interpolation is a windowed form of a Sinc filter which gives better performance than the other interpolation methods [2]. The reviewed traditional interpolation methods do not provide sufficient visual quality particularly around sharp edges due to the use of local smoothness filters [3]. Wavelet based super-resolution techniques in some extents could mitigate the over smoothing problem of the traditional image resolution enhancement techniques by using a set of filter banks.

Most of wavelet based image resolution enhancement techniques are based on the idea that the low resolution image is the baseband of a higher resolution image and then try to estimate the unknown detail coefficients to generate the high resolution image. Zhao et. al [4] proposed a wavelet based image resolution enhancement technique based on wavelets' Hidden Markov Tree (HMT) model. They modelled the dependencies of multiscale wavelet coefficients through the state probabilities of wavelet coefficients by wavelets' HMT. Cycle-spinning technique was further used to suppress the artifacts in the reconstructed high-resolution images. Demirel and Anbarjafari [5] reported a Complex Wavelet Transform (CWT) based image resolution enhancement technique. A dual-tree CWT (DT-CWT) was first applied to the input image. The resolution enhancement was achieved by using directional selectivity provided by the CWT, where the highfrequency subbands in six different directions contribute to the sharpness of the high-frequency details. An inverse DTCWT was finally applied to the coefficients in the highfrequency subbands to generate the super-resolution image. Temizel and Vlachos [6] used wavelet domain zero padding (WZP) and cycle spinning methodology to create high resolution images from low resolution images. A DT-CWT and a Non-Local Means (NLM) based image resolution enhanced technique were reported in [7]. DT-CWT used to generate high frequency subbands. Windowed form of the Sinc filter was then employed to interpolate the highfrequency subbands and the low resolution input image. The NLM filter was then applied to the high frequency subbands to reduce artefacts generated by DT-CWT. Finally, an inverse DT-CWT combines the resulting high frequency subbands and the low resolution input image, generating the super-resolution image. Piao et al. [8] also proposed a 
resolution enhancement method using inter-subband correlation in which the sampling phase in Discrete Wavelet Transform (DWT) was considered. They designed interpolation filters by analysing correlations between subbands having different sampling phases in the lower level, and then applied to the correlated subbands in the higher level, with the assumption that correlations between two DWT subbands in the higher level are similar to that in the lower level. Another wavelet based image resolution enhancement technique was proposed in [9]. The proposed algorithm used a DWT to decompose the input image into its frequency subbands. The resulting frequency subbands were then interpolated using the Bicubic algorithm. The difference between the input low resolution image and the interpolated low frequency subband was then used to refine the interpolated high frequency subbands. An inverse DWT was finally applied to generate the super-resolution image. Temizel and Vlachos proposed another wavelet based image resolution enhancement technique using Cycle Spinning (CS) [10], which outperformed other state of the art techniques, at the time. The authors of this paper reported another wavelet based image resolution enhancement algorithm, which operated in a quad-tree wavelet decomposition framework and exploited wavelet coefficient correlation in a local neighbourhood sense [11]. This method employed linear least-squares regression algorithm to estimate the wavelet high-frequency coefficients and generated superior results compared to the conventional methods for a wide range of test images. Azam et. al [12] reported another image resolution enhancement algorithm based on cycle spinning and stationary wavelet subband padding. The proposed technique used Stationary Wavelet Transformation (SWT) to decompose the low resolution image into frequency subbands. The generated subbands were further interpolated using either bicubic or lanczos interpolation, and these interpolated subbands were put into inverse SWT process for generating intermediate high resolution image. Cycle spinning method was finally applied to reduce blocking artifacts, followed by traditional Laplacian sharpening filter to highlight the sharp edges.

While various wavelet-based image enlargement methods have shown outstanding performance in generating high quality enlarged images, no significant research has been done to identify the best and most appropriate type of wavelets in wavelet based image resolution enhancement techniques. One common problem with most enlargement techniques is zigzagged edges and jagged images following the enlargement [13], which wavelet based image resolution enhancement techniques are known to be a solution for this problem . This paper investigates the effect of different types of wavelet transform chosen from different wavelet families in a generic image resolution enhancement technique to identify the most appropriate wavelet type for image enlargement purpose. The rest of the paper is organized as follows: the proposed system for this investigation is explained in Section 2, experimental results are presented in Section 3 and finally paper will be concluded in Section 4 .

\section{Wavelet Technique \\ based Image Enlargement}

To investigate the efficiency of different types of wavelet transforms from different wavelet families, a wavelet based image enlargement technique presented in [6] titled "wavelet domain image resolution enhancement using cycle-spinning” is employed. This specific method has been implemented in this study as there are various superior image enlargement methods that use similar building blocks. In addition, this algorithm employs the cycle-spinning technique, which is widely used amongst wavelet based image enlargement techniques to boost the quality of the enlarged images. For the purpose of the investigation, different wavelet transforms from wavelet transform families such as Daubechies, symlet, Coiflet, Biorthogonal and Reverse Biorthogonal wavelets are used. Figure 1 shows the block diagram of the proposed image resolution enhancement technique. A low resolution image is input to the system an assumed to be the baseband of a wavelet transformed image where the values of the elements of all high frequency subbands, LH, HL and $\mathrm{HH}$ are set to zero. An Inverse Wavelet Transform (IMWT) block produces a High-Resolution (HR) image. The concept of the cycle spinning technique is used in this stage. The resulting HRimage is first spatially shifted with different $2 \mathrm{D}$ shift values, generating $\mathrm{N}$ Shifted High Resolution (SHR) images. Then the N 2D-shift vectors are determined using Equation (1).

$$
\left[\begin{array}{cccc}
(-k,-k) & (-k,-k+1) & \cdots & (-k, k) \\
(-k+1,-k) & (-k+1,-k+1) & \cdots & (-k+1, k) \\
\cdots & \cdots & \cdots & \cdots \\
(k,-k) & (k,-k+1) & \cdots & (k, k)
\end{array}\right]
$$

where $\mathrm{k}$ is the maximum number of pixel shifts in both horizontal and vertical directions.

In this paper $\mathrm{k}$ was set to 3 , as system exhibits its best performance with this value. Number of shifted images, $\mathrm{N}$, can be calculated using Equation (2):

$$
\mathrm{N}=(2 \mathrm{k}+1)^{2}
$$

Each of the resulting $\mathrm{N}$ Shifted High Resolution (SHR) images is then processed as follows:

I) A 2D Wavelet Transform is applied on the SHR-image, decorrelating the SHR-image into its wavelet subbands;

II) The coefficients in the resulting wavelet high frequency subbands are set to zero;

III) A 2D Inverse Wavelet Transform is used to generate a Primary Spatially Shifted Super Resolution (PSSSR) image from the resulting coefficients;

IV) The PSSSR-image is spatially inverse shifted, bringing back the image coefficients onto its original positions.

The output super-resolution image is created by averaging the resulting $\mathrm{N}$-shift compensated PSSSR-images. 


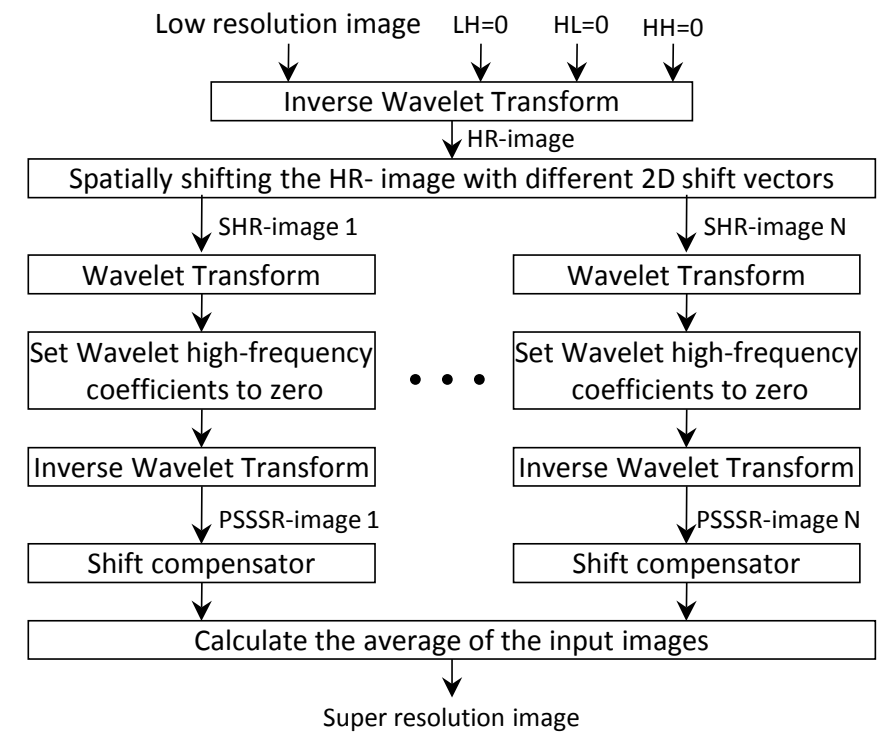

Figure 1: Block diagram of the wavelet-based image enlargement algorithm.

\section{Experimental results}

To evaluate the efficiency of different types of wavelet transforms from the known wavelet families, four standard test images, Lena, Peppers, Mandrill and Barbara, containing a range of textures and representing a diversity of low to high frequency components, were chosen. To generate low resolution replicas of the test images, the input images were filtered using a two dimensional low-pass $5 \times 5$ Blackman filter and then down sampled both horizontally and vertically by the factor of two [14]. The Blackman 2D FIR filter coefficients are tabulated in Table 1 [14].The resulting low resolution images were then enlarged using the wavelet based image enlargement algorithm, where the algorithm uses different wavelets. Different wavelet transforms from wavelet transform families such as Daubechies, Symlets, Coiflet, Biorthogonal, Reverse Biorthogonal and Discrete Meyer wavelets were used in this investigation. In total around 33 different wavelet transform functions were considered for the analysis. The Peak Signal to Noise Ratio (PSNR) measurement and the Structural SIMilarity (SSIM) index, which has been proven to be consistent with the properties of human virtual system, were chosen to assess the quality of the enlarged images against their corresponding ground-truth images (the original test images were assumed to be the ground truth images). The PSNR measurements and the SSIM indexes for the enlarged images are tabulated in Table 2 and 3 , respectively. From Table 2, it is observed that in the case of Lena and pepper test images, which contain a mixture of low and high frequency components (medium details), Daubechies family wavelet Daubechi 9/7 and LeGall 5/3

\begin{tabular}{|l|l|l|}
\hline 0.0381 & 0.1051 & 0.0381 \\
\hline 0.1051 & 0.4273 & 0.1051 \\
\hline 0.0381 & 0.1051 & 0.0381 \\
\hline
\end{tabular}

Table 1: The Blackman 2D FIR filter coefficients [14].

\begin{tabular}{|c|c|c|c|c|}
\hline \multirow[b]{2}{*}{ Wavelet Types } & \multicolumn{4}{|c|}{$\begin{array}{l}\text { PSNR } \\
\end{array}$} \\
\hline & Lena & Peppers & Mandrill & Barbara \\
\hline \multicolumn{5}{|c|}{ Discrete Meyer } \\
\hline dmey & 21.95 & 22.17 & 19.2 & 19.86 \\
\hline \multicolumn{5}{|c|}{ Daubechies } \\
\hline Daubechi 9/7 & 33.75 & 32.79 & 30.17 & 24.86 \\
\hline LeGall 5/3 & 32.06 & 31.78 & 27.56 & 24.63 \\
\hline db1 (Haar) & 29.83 & 29.88 & 25.87 & 24.07 \\
\hline db5 & 22.96 & 22.22 & 21.79 & 21.00 \\
\hline \multicolumn{5}{|c|}{ Biorthogonal } \\
\hline bior1.3 & 21.20 & 21.25 & 19.23 & 19.52 \\
\hline bior1.5 & 21.15 & 21.2 & 19.13 & 19.48 \\
\hline bior2.4 & 22.40 & 22.49 & 20.00 & 20.24 \\
\hline bior2.8 & 22.38 & 22.46 & 19.97 & 20.22 \\
\hline bior3.3 & 24.26 & 24.39 & 21.42 & 21.39 \\
\hline bior3.7 & 24.23 & 24.37 & 21.38 & 21.37 \\
\hline bior4.4 & 21.96 & 22.19 & 19.39 & 19.93 \\
\hline bior5.5 & 25.60 & 26.09 & 21.99 & 21.80 \\
\hline bior6.8 & 22.06 & 22.26 & 19.44 & 19.99 \\
\hline \multicolumn{5}{|c|}{ Reverse Biorthogonal } \\
\hline rbio1.3 & 21.00 & 21.08 & 18.95 & 19.35 \\
\hline rbio1.5 & 20.86 & 20.97 & 18.71 & 19.24 \\
\hline rbio2.4 & 21.75 & 22.05 & 18.97 & 19.75 \\
\hline rbio2.8 & 21.59 & 21.93 & 18.67 & 19.58 \\
\hline rbio3.3 & 22.71 & 23.19 & 19.18 & 19.95 \\
\hline rbio3.7 & 22.61 & 23.18 & 18.90 & 19.77 \\
\hline rbio4.4 & 22.21 & 22.39 & 19.62 & 20.09 \\
\hline rbio5.5 & 26.33 & 26.4 & 23.04 & 22.40 \\
\hline rbio6.8 & 22.00 & 22.22 & 19.31 & 19.92 \\
\hline \multicolumn{5}{|c|}{ Coiflets } \\
\hline coif2 & 28.12 & 26.99 & 26.75 & 23.6 \\
\hline coif3 & 25.13 & 24.14 & 23.99 & 22.12 \\
\hline coif5 & 19.62 & 19.35 & 17.86 & 18.6 \\
\hline \multicolumn{5}{|c|}{ Symlets } \\
\hline sym2 & 28.13 & 23.13 & 28.50 & 28.03 \\
\hline sym3 & 27.21 & 22.85 & 22.40 & 21.74 \\
\hline sym10 & 22.55 & 22.83 & 19.49 & 20.29 \\
\hline sym15 & 23.03 & 23.18 & 20.26 & 20.48 \\
\hline sym20 & 26.22 & 26.50 & 22.68 & 22.28 \\
\hline sym30 & 26.32 & 26.55 & 22.76 & 22.30 \\
\hline
\end{tabular}

Table 2: The PSNR comparisons for different wavelets from wavelet transform families.

produce the highest PSNR. In case of Mandrill and Barbara test images, which contain a high range of textures and high frequency components, Symlet family wavelet Sym2 and Daubechies family wavelet Daubechi 9/7produce the highest PSNR. This implies that wavelets with less vanishing moments give less smoothing and remove fewer details, but wavelets with more vanishing moments produce more distortions. The presented results in Table 2 show that, the performance of the image enlargement technique is a function of the chosen wavelet as well as the frequency components or 


\begin{tabular}{|c|c|c|c|c|}
\hline \multirow[b]{2}{*}{ Wavelet Types } & \multicolumn{4}{|c|}{ SSIM } \\
\hline & Lena & Peppers & Mandrill & Barbara \\
\hline \multicolumn{5}{|c|}{ Discrete Meyer } \\
\hline dmey & 0.71 & 0.78 & 0.45 & 0.65 \\
\hline \multicolumn{5}{|c|}{ Daubechies } \\
\hline Daubechi 9/7 & 0.98 & 0.97 & 0.97 & 0.89 \\
\hline LeGall 5/3 & 0.97 & 0.97 & 0.92 & 0.87 \\
\hline db1 (Haar) & 0.94 & 0.96 & 0.89 & 0.87 \\
\hline $\mathrm{db} 5$ & 0.92 & 0.92 & 0.85 & 0.82 \\
\hline \multicolumn{5}{|c|}{ Biorthogonal } \\
\hline bior1.3 & 0.66 & 0.73 & 0.45 & 0.6 \\
\hline bior1.5 & 0.66 & 0.73 & 0.43 & 0.6 \\
\hline bior2.4 & 0.73 & 0.79 & 0.46 & 0.66 \\
\hline bior2.8 & 0.73 & 0.79 & 0.46 & 0.66 \\
\hline bior3.3 & 0.82 & 0.86 & 0.61 & 0.74 \\
\hline bior3.7 & 0.82 & 0.76 & 0.61 & 0.74 \\
\hline bior4.4 & 0.72 & 0.78 & 0.43 & 0.65 \\
\hline bior5.5 & 0.88 & 0.91 & 0.77 & 0.82 \\
\hline bior6.8 & 0.72 & 0.79 & 0.42 & 0.65 \\
\hline \multicolumn{5}{|c|}{ Reverse Biorthogonal } \\
\hline rbio1.3 & 0.66 & 0.72 & 0.33 & 0.52 \\
\hline rbio1.5 & 0.68 & 0.72 & 0.33 & 0.59 \\
\hline rbio2.4 & 0.71 & 0.78 & 0.44 & 0.64 \\
\hline rbio2.8 & 0.70 & 0.77 & 0.39 & 0.63 \\
\hline rbio3.3 & 0.79 & 0.84 & 0.57 & 0.72 \\
\hline rbio3.7 & 0.78 & 0.83 & 0.55 & 0.71 \\
\hline rbio4.4 & 0.72 & 0.79 & 0.44 & 0.65 \\
\hline rbio5.5 & 0.79 & 0.92 & 0.77 & 0.82 \\
\hline rbio6.8 & 0.72 & 0.78 & 0.42 & 0.64 \\
\hline \multicolumn{5}{|c|}{ Coiflets } \\
\hline coif2 & 0.96 & 0.97 & 0.95 & 0.9 \\
\hline coif3 & 0.93 & 0.9 & 0.9 & 0.88 \\
\hline coif5 & 0.7 & 0.77 & 0.6 & 0.64 \\
\hline \multicolumn{5}{|c|}{ Symlets } \\
\hline sym2 & 0.94 & 0.87 & 0.88 & 0.95 \\
\hline sym3 & 0.91 & 0.83 & 0.80 & 0.84 \\
\hline sym10 & 0.74 & 0.84 & 0.44 & 0.66 \\
\hline sym15 & 0.79 & 0.84 & 0.58 & 0.73 \\
\hline sym20 & 0.87 & 0.92 & 0.77 & 0.83 \\
\hline sym30 & 0.88 & 0.93 & 0.78 & 0.8 \\
\hline
\end{tabular}

To give a visual perception of the resulting enlarged images, a section of the original Lena, Pepper, Mandrill and Barbara test images including the low resolution images, and the enlarged images using Daubechi 9/7, Coif5, Sym10, Dmey, Bior6.8, rbio5.5 are shown in Figure $2,3,4$ and 5 respectively. It can be seen that the proposed technique when using Daubechi 9/7 and Sym2 produces superior results with high contrast and less pixilation in smooth areas better edges preservation.

\section{Conclusions}

This paper investigated the performance of a generic wellknown wavelet and cycle-spinning based image resolution enhancement method with respect to the choice of wavelets from different wavelet transform families. Different wavelet transforms from wavelet transform families such as Daubechies, symlet, Coiflet, Biorthogonal, Reverse Biorthogonal and Discrete Meyer wavelets were chosen in this investigation. Results indicated that that the enlargement method produces superior results using Daubechi 9/7 and Sym2 when enlarging images containing significantly higher frequency components. Images contain a mixture of both low and high resolution exhibit superior results when Daubechi 9/7 employed. The presented visual quality for the four test images also confirm the superior visual quality of the enhancement technique using Daubechies family and Symlets family. The generated results showed that the performance of the image enlargement technique is a function of the chosen wavelet and also texture and frequency components of the input image to achieve a desired quality for the enlarged image.

\section{References}

[1] R. C. Gonzalez, R. E. Woods, "Digital image processing, 3rd ed., Englewood Cliffs, NJ: Prentice-Hall, pp. 87-89, (2008).

[2] A. S. Glassner, K. Turkowski, S. Gabriel, "Filters for common resampling tasks" Graphics Gems, New York: Academic, pp. 147-165, (1990).

[3] W.K. Carey, D.B. Chuang, S. S.Hemami, "Regularity preserving Image Interpolation”, IEEE Trans. on Image Process, volume 8, pp.1295-1297, (1999).

[4] S. Zhao, H. Han, S. Peng, "Wavelet-domain HMT-based

Table 3: The SSIM comparisons for different wavelet transform.

texture of the image. However, it is well known that the PSNR is not a reliable metric to judge the visual quality of the enlarged images and the human eyes are the final judges for assessing the quality of the images. Hence, the SSIM index, which is consistent with the perception of the human eyes, is a more reliable metric to be used for evaluating the quality of the enlarged images. Table 3 shows the results of the SSIM metric for the four test images using the image enlargement method. The SSIM metric further confirms that Daubechies family and Symlets family generally achieved the highest visual perception amongst other wavelet types. (2003).

[5] A. Temizel and T. Vlachos, "Image resolution up-scaling in the wavelet domain using directional cycle spinning”, Journal of Electronic Imaging, volume 14, pp.1-3, (2005).

[6] A. Temizel and T. Vlachos, "Wavelet Domain Image Resolution Enhancement using Cycle-spinning”, Electronics Letters, volume 41, pp. 119-121, (2005). 


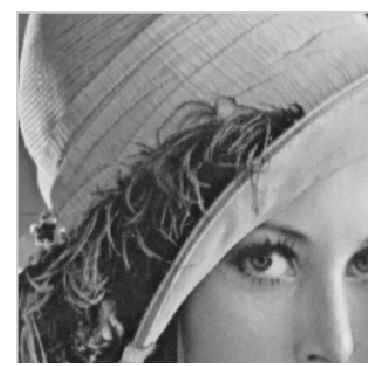

a) Ground truth

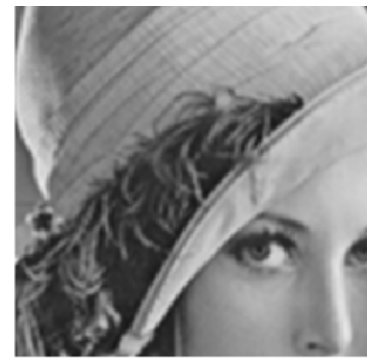

c) daubechi $9 / 7$

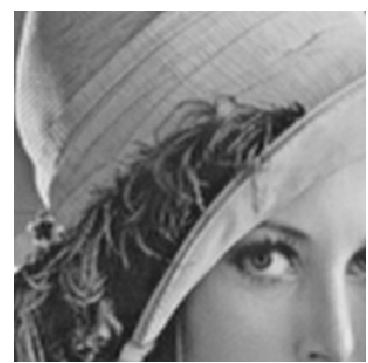

e) dmey

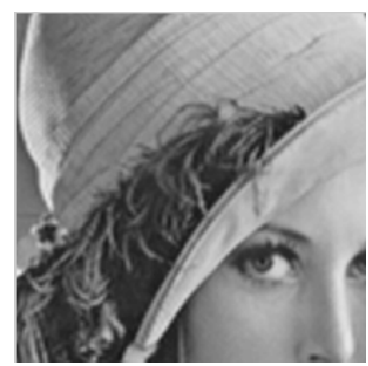

g) bior6.8

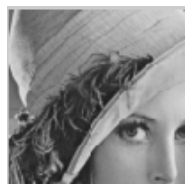

b) Low resolution

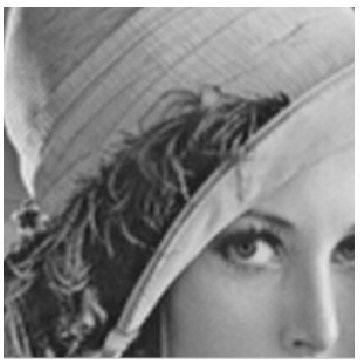

d) coif5

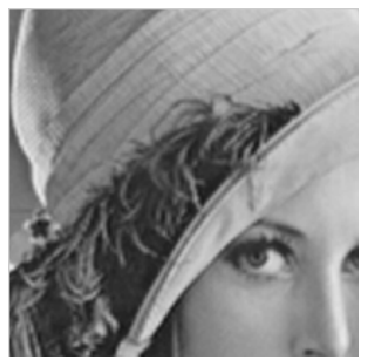

f) $\operatorname{sym} 10$

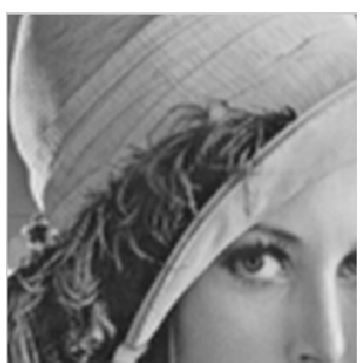

h) rbio5.5

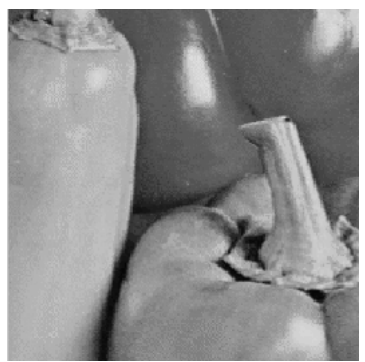

a) Ground truth

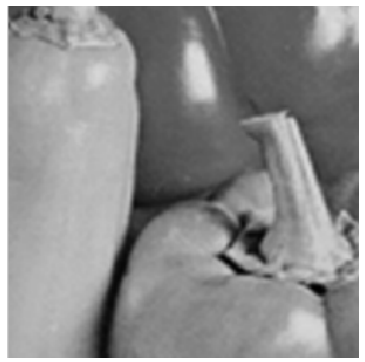

c) daubechi $9 / 7$

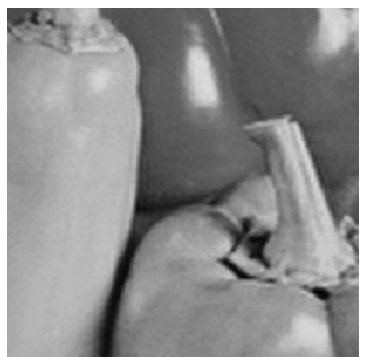

e) dmey

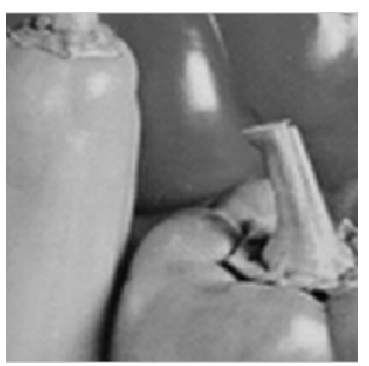

g) bior6.8

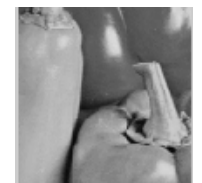

b) Low resolution

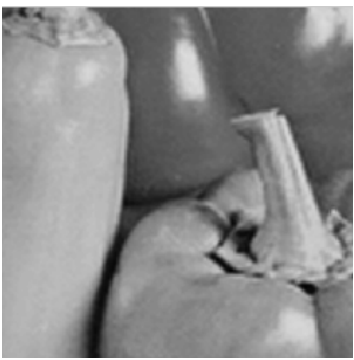

d) coif5

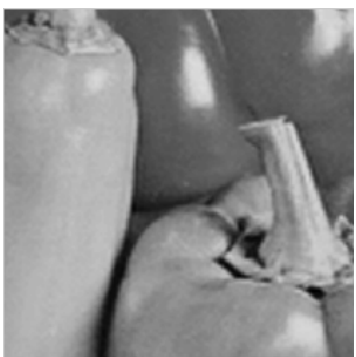

f) $\operatorname{sym} 10$

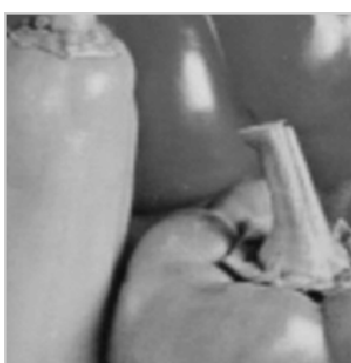

h) rbio5.5

Figure 2: A section of Lena test image: a) Ground truth image b) low resolution image and c) - h) enlarged images using different wavelets.

[7] A. Temizel and T. Vlachos, "Wavelet Domain Image Resolution Enhancement," IEE Proc. Vis. Image Signal Processing, volume 153, pp. 25-30, (2006).

[8] Y. Piao, I. Shin, H. Park, H, "Image resolution enhancement using inter-subband correlation in wavelet domain”, ICIP2007, volume 1, pp.445-448, (2007).

[9] H. Demirel, G. Anbarjafari, "Satellite image resolution enhancement using complex wavelet transform”, IEEE Geoscience and Remote Sensing Letters, volume 7, pp. 123126, (2010).

Figure 3: A section of Pepper test image: a) Ground truth image b) low resolution image and c) - h) enlarged images using different wavelets.

[10] H. Demirel, G. Anbarjafari, "Image resolution enhancement by using discrete and stationary wavelet decomposition”, IEEE Transaction on Image Processing, volume 20, pp. 1458-1460, (2011).

[11] M. Z. Iqbal, A. Ghafoor, A. M. Siddiqui, "Satellite Image Resolution Enhancement Using Dual-Tree Complex Wavelet Transform and Nonlocal Means”, IEEE Geoscience and Remote Sensing Letters, volume 10, pp. 451-455, (2013). 


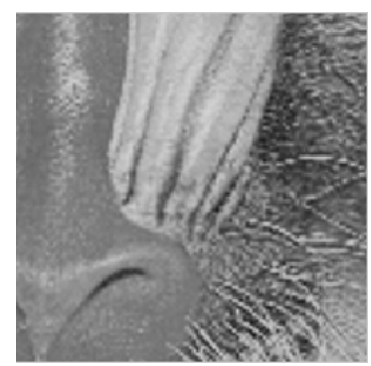

a) Ground truth

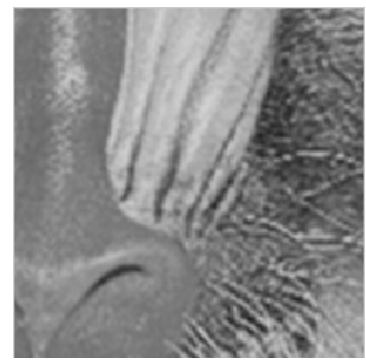

c) daubechi $9 / 7$

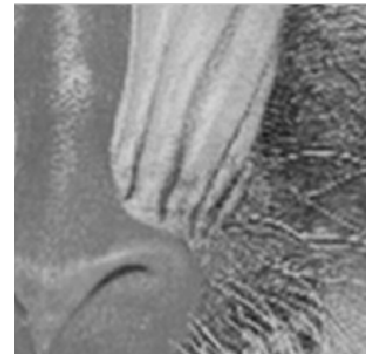

e) dmey

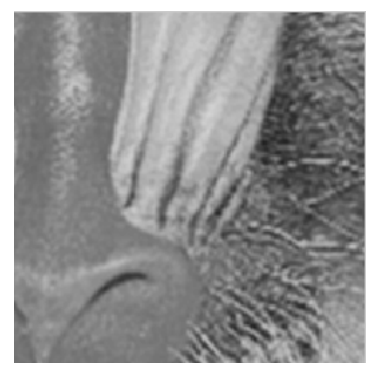

g) bior6.8

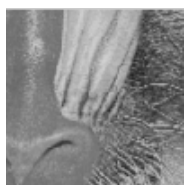

b) Low resolution

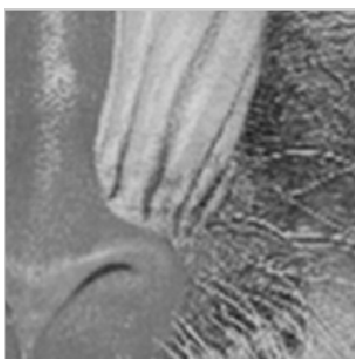

d) coif5

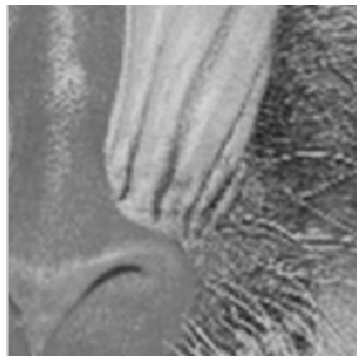

f) $\operatorname{sym} 10$

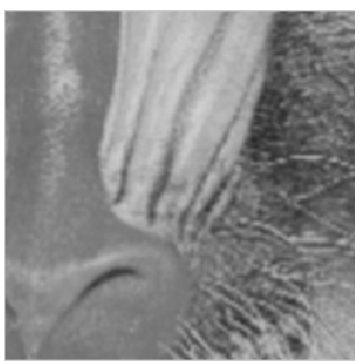

h) rbio5.5

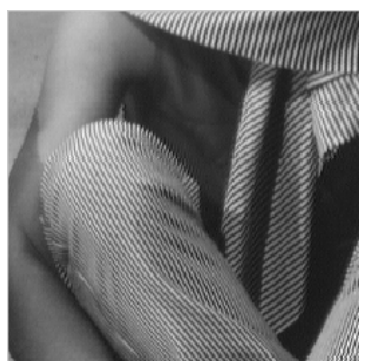

a) Ground truth

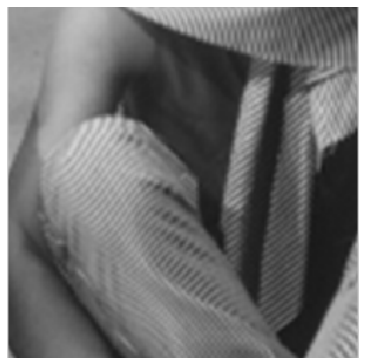

c) daubechi $9 / 7$

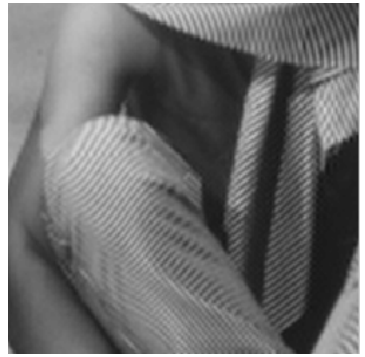

e) dmey

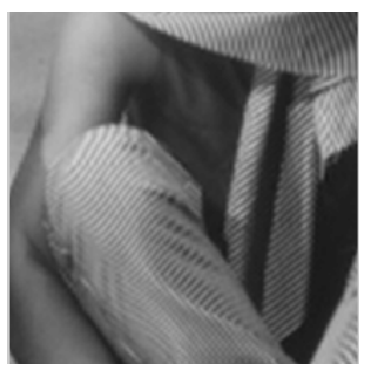

g) bior6.8

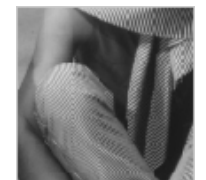

b) Low resolution

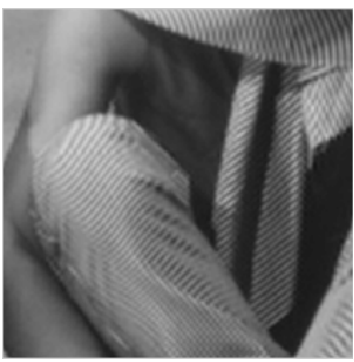

d) coif5

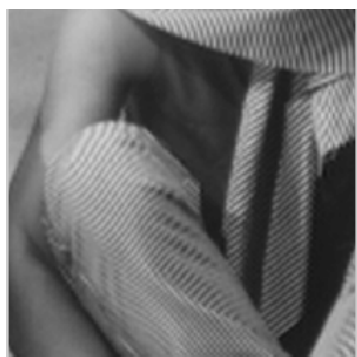

f) $\operatorname{sym} 10$

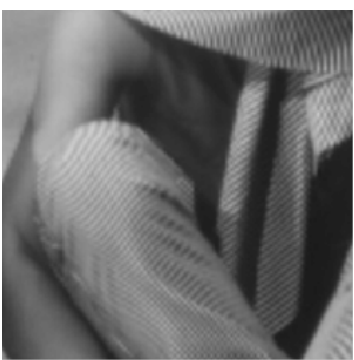

h) rbio5.5

Figure 4: A section of Mandrill test image: a) Ground truth image b) low resolution image and c) - h) enlarged images using different wavelets

[12] S. Azam, F. T. Zohra, M. M. Islam, "Remote Sensing Image Resolution Enlargement Algorithm Based on Wavelet Transformation”, International Journal on Image, Graphics and Signal Processing, volume 6, pp.19-26, (2014).

[13] H-Y. Lin, C-Y. Lin, C-J. Lin, C-S. Yang, C-Y. Yu, “A Study of Digital Image Enlargement and Enhancement”, Mathematical Problems in Engineering, volume 2014, pp. 17, (2014).

Figure 5: A section of Barbara test image: a) Ground truth image b) low resolution image and c) - h) enlarged images using different wavelets.

[14] P. Bagheri Zadeh and A. Sheikh Akbari, "Image Resolution Enhancement using Multi-wavelet and CycleSpinning”, The UKACC (United Kingdom Automatic Control Council) International Conference on Control (CONTROL 2012), pp. 789 - 792, (2012). 